\title{
Memory conjunction errors for realistic faces are consistent with configural processing
}

\author{
ELINOR MCKONE and YU XI PEH \\ Australian National University, Canberra, Australia
}

\begin{abstract}
Conjunction faces are formed from feature sets learned across different faces. In previous studies, false alarms ("old" responses) to conjunctions have been very high, approaching hits to old faces; this is surprising, because, perceptually, upright faces are processed configurally, with strong integration of parts into the whole. We test the idea that the atypical reliance on unrelated parts could be due to using unnatural line drawings as stimuli, and to forming conjunctions across external features (e.g., hair) and internal features (e.g., eyes, mouth). We used realistic face stimuli and conjunctions made entirely from internal features. Results were, as expected, consistent with configural processing for upright faces (hits to old faces much greater than FA to conjunctions) and not for inverted faces (hits to old $=$ FA to conjunctions).
\end{abstract}

Memory conjunction errors (Reinitz, Lammers, \& Cochran, 1992) are a false memory phenomenon in which people miscombine parts of separate experiences and remember a conjunction that does not accurately correspond to any single previously experienced event. For example, after studying HANDSTAND and SHOTGUN, there is a tendency to say "old" to HANDGUN in a subsequent recognition test.

Conjunction errors can be used to assess the extent to which subjects' performance is sensitive to study-test match in the whole item (HANDSTAND), as opposed to study-test match in only component parts (HAND, STAND). If subjects are sensitive to the particular pairings of components seen at study, truly old stimuli will be judged as old more often than conjunction stimuli, despite the conjunction stimuli being composed entirely of old parts; that is, a percentageof-"old"-responses measure would show the pattern old > conjunction. If subjects are also sensitive to matches in local components, conjunction stimuli, in having familiar parts, should still be (falsely) recognized as old more often than entirely new items; that is, conjunction $>$ new. Two extreme outcomes are then possible. If performance is based entirely on matching the whole studied item (e.g., because no part decomposition takes place in either perception or memory), the pattern old $>$ conjunction $=$ new will be obtained. Alternatively, if performance is based purely on part matches (e.g., because there is no binding of parts into wholes), the pattern will be old = conjunction $>$ new.

This research was supported by Australian Research Council Grant DP0208630. Stefan Horarik and Jacqui Brewer assisted in subject testing and data analysis. We thank Gillian Rhodes, Michael Wenger, and an anonymous reviewer for comments on the manuscript. Correspondence should be addressed to E. McKone, School of Psychology, Australian National University, Canberra, ACT 0200, Australia (e-mail: elinor .mckone@anu.edu.au).
Our interest here is in memory conjunction errors for faces. Memory errors have been observed using conjunctions made of the hair and mouth of one studied face with the eyes and nose of another (Reinitz et al., 1992), or the hair and chin of one face with the internal face features of another (Searcy, Bartlett, \& Memon, 1999). In these studies, false alarms (FAs) to conjunctions have been very high: In many cases, the conjunction condition lies much closer to the old condition than the new condition (e.g., old $=79 \%$ "old," conjunction $=68 \%$ "old," new $=15 \%$ "old"; Reinitz \& Hannigan, 2001, Experiment 3), and, in one study, the FA rate for conjunction faces and the hit rate for old faces were actually equal (Hannigan \& Reinitz, 2000 , Experiment 2). These results indicate weak or no sensitivity to any relationship between face parts.

In current theories of memory conjunction errors (Jones \& Jacoby, 2001; Reinitz \& Hannigan, 2001; Rubin, Van Petten, Glisky, \& Newberg, 1999), these results have been treated as unsurprising, with a general presumption that conjunction errors for faces can be explained on the same basis as conjunction errors for words, sentences, or any other stimulus type. From the perspective of the literature on face perception, however, they appear rather peculiar.

The reason is that the stimuli in these experiments were upright faces. Under most circumstances, perception of upright faces is not based on unrelated parts, but is instead configural/holistic, involving strong integration of parts into wholes. Many paradigms have confirmed this finding (for a review, see Maurer, Le Grand, \& Mondloch, 2002). Here, we mention only two, chosen because their methods bear closest resemblance to the memory conjunction procedure. First, in Young, Hellawell, and Hay's (1987) aligned versus misaligned composite paradigm, halves of different famous faces (e.g., a modern example would be the top half of George Bush with the bottom half of Tony Blair) were presented together either physically aligned or offset by half a face width. For upright faces, reaction 
times to name one half (e.g., "George Bush") were slowed by approximately $200 \mathrm{msec}$ in the aligned condition. This indicates integration of halves into a new whole and corresponds to observers' perceptions that the composite seems to be a new person. Second, L. B. Cohen and Cashon (2001) habituated infants to two faces and then showed a conjunction face made of the internal features of one with the external features of the other. For upright stimuli, infants showed strong dishabituation, indicating they treated the conjunction face as new, not as old.

Importantly, this configural/holistic processing occurs only for upright faces. When the same stimuli are shown inverted (upside down), perception is based on unrelated parts: For example, aligned-half and unaligned-half conditions did not differ in Young et al. (1987), and there was no dishabituation to the conjunction face in L. B. Cohen and Cashon (2001). These behavioral results are consistent with neuroimaging evidence (see, e.g., Kanwisher, McDermott, $\&$ Chun, 1997) and neuropsychological evidence (see, e.g., Moscovitch, Winocur, \& Behrmann, 1997) that different systems support perception of upright faces, on the one hand, and inverted faces (and objects), on the other.

Our aim in the present study was to resolve the apparent conflict between the previous memory conjunction results for upright faces (indicating little sensitivity to the relationship between parts) and the results just described (showing configural processing for upright faces). We suggest that several aspects of the memory conjunction studies could have produced an atypically strong reliance on independent parts. Most obviously, Reinitz's experiments (Hannigan \& Reinitz, 2000; Reinitz \& Hannigan, 2001; Reinitz et al., 1992) used schematic line drawings showing quite unnatural representations of faces, potentially encouraging attention to parts. Second, Searcy et al. (1999) used real photographs, but their 1950s yearbook stimuli, like Reinitz's line drawings, contained unusual and highly salient hairstyles. Hair cues can be used by prosopagnosics to "recognize" faces (Buxbaum, Glosser, \& Coslett, 1999), suggesting that hair is processed by the (part-based) objectrecognition system rather than the true face-processing system. Third, hair has always been one of the features used to construct the conjunction faces (e.g., hair-mouth with eyes-nose in Reinitz's experiments). If hair and internal face features are processed by two different perceptual systems, it could be quite reasonable that this particular combination of feature sets is represented independently in memory. Whether the same would remain true for conjunctions made of entirely internal face features, however, is an open question. Fourth, most previous studies have presented faces in pairs at study; this was done to allow conjunctions derived from simultaneously learned faces to be compared with conjunctions derived from faces learned on different trials, but might have led to unusually strong attention to parts by encouraging a feature-byfeature comparison strategy at encoding.

In the present study, we reexamined memory conjunction errors for faces, with important changes to the stimuli. Faces (Figure 1) were natural-appearing composite photographs with no hair. Conjunction stimuli were made entirely from internal face features by combining the eyes-eyebrows region of one studied face with the nosemouth region of another. These feature combinations were chosen because, in free scanning tasks, subjects fixate more on the eyes than on either the nose or the mouth; thus, using the nose and mouth together should produce reasonably equal attention weight to the two feature sets.

If our interpretation of Reinitz's results is correct, we predict that, in the upright orientation, our new stimuli should produce memory performance that is highly sensitive to the relationship between parts that was seen at study; that is, "old" responses to old should now be substantially greater than to conjunction. We also expect conjunction $>$ new, given that memory for isolated face parts is above chance in previous studies (see, e.g., Tanaka \& Farah, 1993). Inverted faces were tested to provide an important comparison. If, as we have suggested, perceptual processing is an important predictor of memory conjunction errors, inverted faces should show a different pattern than do upright faces. Specifically, given the evidence of no configural processing for inverted faces, we would predict old $=$ conjunction $>$ new .

\section{EXPERIMENT 1}

Although we used stimuli that were different from those previously used by Reinitz, we wanted to begin by otherwise retaining his most commonly used procedure. Thus, in Experiment 1, faces were learned in pairs (Reinitz et al., 1992), and conjunction test faces were made of parts seen simultaneously or nonsimultaneously at study.

\section{Method}

Subjects and Design. The subjects were forty-eight Caucasian Australian undergraduates of the Australian National University, reporting normal vision. Most received course credit. Twenty-four studied and were tested on upright faces; 24 studied and were tested on inverted faces. Memory condition (old, simultaneous conjunction [SC], nonsimultaneous conjunction [NSC], and new) was varied within subjects. To avoid floor effects on memory, the stimuli were presented in two study-test cycles, each consisting of a study phase, a distractor phase, and a test phase. The dependent measure was the percentage of "old" responses in old-new recognition.

Materials. All stimuli were composite faces, formed by pasting the eyes and eyebrows of one real individual, and the nose and mouth of another, onto a generic background head. A given subject never saw a particular feature set in more than one test face (although obviously this feature set may also have been seen at study).

Original faces came from the Harvard Vision Lab Face Database (Tong \& Nakayama, 1999) and the University of Stirling Psychological Image Collection. Faces were ethnically similar to Caucasian Australians. Fifty-six real individuals were used to create 112 composite faces. Fifty-six eye-eyebrow sets were randomly ordered (E1-E56), and 56 nose-mouth sets were randomly ordered (N1N56). Four completely crossed composites were then formed from each of the two sets, e.g., for the first two, the composites E1-N1, E2-N2, E1-N2, and E2-N1 were created. Sets from the same original face were never put together.

Of the 112 composite faces, 96 were target stimuli (study or test faces) and 16 were fillers. Each of the two study phases contained 13 trials (each presenting a pair of faces): 2 primacy filler trials, 9 target trials, and then 2 recency filler trials. At test, old faces were exact repeats of a composite seen at study. New faces had neither feature set 


\section{Composite Face Stimuli}

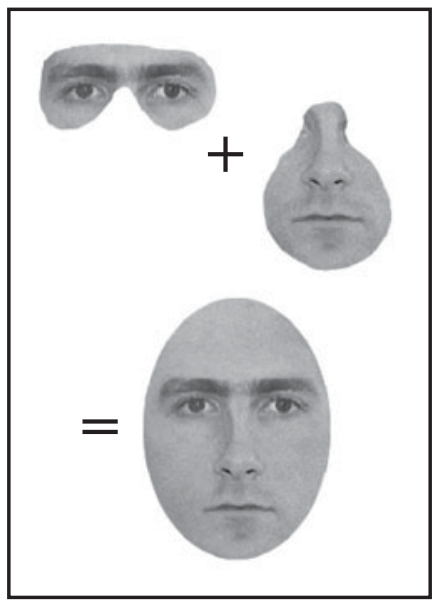

Formulation of Conjunction Test Faces

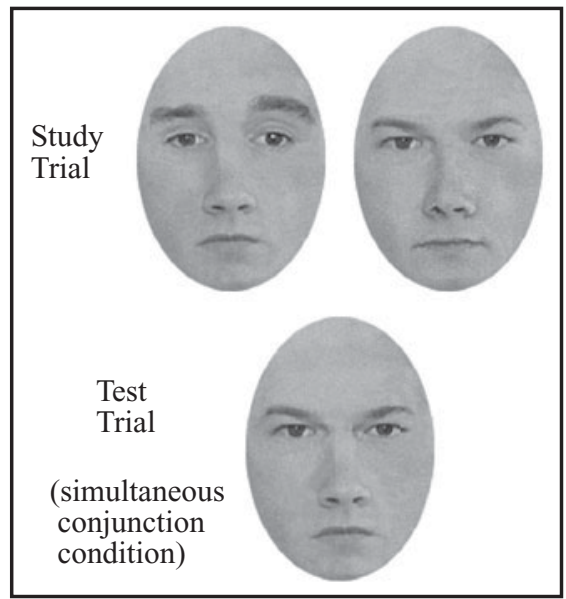

Figure 1. All stimuli were composite faces, formed by pasting the eyes-eyebrows of one real face and the nose-mouth of another onto a generic background head. Conjunction test faces combined feature sets across two studied faces; in Experiment 1, faces were learned in pairs, and simultaneous conjunction condition items were made between composites from the same study trial.

(eyes-eyebrows or nose-mouth) appear at study. Conjunction faces had both feature sets appear at study, but in different faces. To use items efficiently, target study pairs were organized in fixed-order triplets (study trials $n, n+1$, and $n+2$ ). Two old test faces were obtained from one item on study trial $n$, and one on trial $n+2$. Two SC test faces were derived from the two items on study trial $n+1$. Two NSC test faces were derived from the unused items on study trial $n$ and $n+2$. Six such triplets (three triplets per study cycle) produced 12 stimuli per test condition. Test items were in a new random order for each subject. Four versions of the memory lists were created that rotated test items through the old, new, SC, and NSC conditions, so that any given item appeared equally often in all conditions.

Procedure. The subjects were tested individually for $1 \mathrm{~h}$. PsyScope software (J. D. Cohen, MacWhinney, Flatt, \& Provost, 1993) displayed faces $\left(3.2^{\circ} \times 2.1^{\circ}\right.$ at $\left.50 \mathrm{~cm}\right)$ on an iMac computer.

At study, each pair of faces was presented side by side for $10 \mathrm{sec}$, with a 10 -sec blank intertrial interval. The subjects were instructed to learn both faces for a later test, dividing learning time equally. The 15-min distractor phase presented nonface tasks (word completion, anagrams, written arithmetic). The test phase presented faces one at a time. The subjects entered "z" for old (i.e., they thought they had seen the face at study) or "l" for new (they thought they had not seen the face at study). No mention was made of the inclusion of conjunction stimuli.

\section{Results}

Mean percentage of "old" responses reflects hits for old faces, and FAs for the other memory conditions. Memory performance (Figure 2; left panel) was well above chance, with the hit rate for truly old faces substantially higher than the FA rate for completely new faces, both upright $[t(23)=6.23, p<.001]$ and inverted $[t(23)=4.12, p<$ $.001]$. Signal detection analysis for old versus new showed that responses were unbiased, with $c$ (Macmillan \& Creelman, 1991) not different from zero either upright $[M=$ $-0.11, S E M=0.08, t(24)=1.33, p>.1]$ or inverted $[M=-.09, S E M=.08, t(24)=1.16, p>.2]$.
For data in Figure 2, a 2 (orientation) $\times 4$ (memory condition) mixed ANOVA revealed a significant two-way interaction [Wilks's $\Lambda=.797 ; F(3,44)=3.74, p<.05$ ], indicating different patterns of conjunction effects for upright and inverted faces. For upright faces, old responses were higher for old faces than for both conjunction conditions: moderately so for SC $[t(23)=3.50, p<.01]$ and very substantially so for NSC $[t(23)=5.15, p<.001]$. This is as would be predicted from face perception studies showing the importance of relationships between parts for upright faces (i.e., configural processing). Old responses were also greater for conjunctions than for entirely new faces $[\mathrm{SC}, t(23)=4.73, p<.001 ; \mathrm{NSC}, t(23)=2.47$, $p<.05]$. This indicates some memory for local feature sets, also consistent with previous face literature (see, e.g., Tanaka \& Farah, 1993). Overall, upright face stimuli were judged as old to some extent if their individual components were old, and more so again if the pairing relationship between these components was also old.

For inverted faces, in contrast, the conjunction conditions lie close to the old condition, arguing for no sensitivity to the pairings between face parts seen at study. Planned $t$ tests confirmed no difference between old and SC (indeed, SC was numerically higher) $[t(23)<1]$ and no difference between old and NSC $[t(23)=1.57, p>.1]$. As expected, both conjunction conditions were substantially above new $[\mathrm{SC}, t(23)=5.75, p<.001$; NSC, $t(23)=$ $4.59, p<.001]$, indicating strong memory for local components. Overall, these results are as would be predicted by perception studies showing part-based rather than configural processing for inverted faces.

A final analysis compared memory in the SC and NSC conditions. FA rates were higher in the former case for both upright faces $[t(23)=3.60, p<.01]$ and inverted 

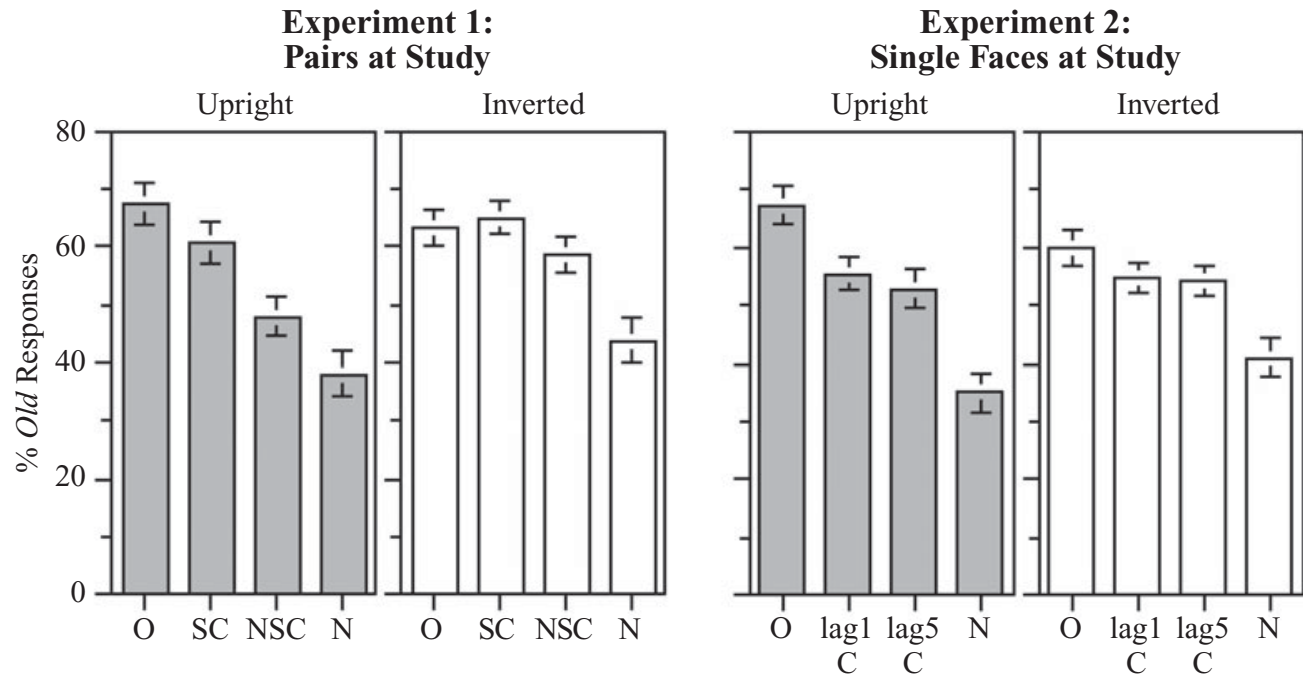

Figure 2. Percentage "old" responses in old-new recognition. Experiment 1: O, old faces; SC, simultaneous conjunction; NSC, nonsimultaneous conjunction; N, new. Experiment 2: lag1C, lag 1 conjunction; lag5C, lag 5 conjunction. Error bars show \pm 1 SEM.

faces $[t(23)=2.39, p<.05]$. We will discuss this result in the General Discussion section.

\section{EXPERIMENT 2}

With the use of natural photographs and the exclusion of hair, the relative contribution of the relationship between face parts was much stronger (for upright faces) in Experiment 1 than in Reinitz's previous studies. One remaining factor from Reinitz's standard procedure that could encourage unusually strong attention to parts was the simultaneous presentation of face pairs at study. In Experiment 2, we examined one-at-a-time presentation (the usual procedure used in face-recognition studies), to see whether the contribution of relationships between parts might further increase.

\footnotetext{
Method

Subjects and Design. Sixty new subjects $(n=30$ upright; $n=$ 30 inverted) were selected from the same pool as were the subjects in Experiment 1. Some were paid \$10. Memory condition (old, lag 1 conjunction, lag 5 conjunction, new) was varied within subjects.

Materials and Procedure. Study lists of Experiment 1 were converted to sequential presentation by moving the face originally appearing on the left-hand side of the screen to the center, and moving the face originally appearing on the right to the next trial. This converted items previously in the SC condition to lag 1 conjunction items (the two feature sets joined together at test had appeared on trials $n$ and $n+1$ at study), and items previously in the NSC condition to lag 5 conjunction items (the two feature sets joined together at test had appeared on trials $n$ and $n+5$ at study).

The resulting 26 single-item study trials per cycle (cf. 13 pairs in Experiment 1) were presented for $4 \mathrm{sec}$ each, approximately equating study time per item to Experiment 1 . The interstimulus interval was $1 \mathrm{sec}$, reducing the total study time to $2.2 \mathrm{~min}$ per cycle (4.3 min in Experiment 1). Otherwise, Experiment 2 was the same as Experiment 1.
}

\section{Results}

Memory (Figure 2; right panel) was again well above chance, with hits for old faces greater than FAs for new faces, both upright $[t(29)=7.22, p<.001]$ and inverted $[t(29)=5.22, p<.001]$. Responses were again unbiased, with $c$ for old versus new not different from zero, either upright $(M=-0.04, S E M=0.08, t<1)$ or inverted $(M=0.0004, S E M=0.08, t<1)$.

In a 2 (orientation) $\times 4$ (memory condition) ANOVA, the interaction with inversion only approached significance $\left[F(3,174)=2.35, M S_{\mathrm{e}}=196.89, p=.075\right]$, but note that ANOVA does not recognize that the memory conditions are ordered in any way (i.e., by physical overlap between study and test). Because one would always expect old to produce the highest \%"old" scores, and new the lowest, with the conjunction conditions ranging somewhere between these two, we conducted a more powerful test of a memory condition $\times$ orientation interaction via trend analysis, coding old, $\operatorname{lag} 1, \operatorname{lag} 5$, and new as ordered, equally spaced conditions. With this coding, an interaction between orientation and the "linear" trend across memory conditions formally indicates some difference in monotonic pattern between upright and inverted. Such an interaction was obtained $\left[F(1,58)=5.892, M S_{\mathrm{e}}=\right.$ $223.89, p<.02]$.

Results for upright and inverted faces considered separately replicated the key findings of Experiment 1 . For upright faces, old was greater than each conjunction condition [lag 1, $t(29)=3.072, p<.01 ; \operatorname{lag} 5, t(29)=3.45$, $p<.005]$, and each conjunction condition was above new [lag 1, $t(29)=6.08, p<.001 ; \operatorname{lag} 5, t(29)=5.66$, $p<.001]$. For inverted faces, neither conjunction condition differed significantly from old $[\operatorname{lag} 1, t(29)=1.46$, $p>.1 ; \operatorname{lag} 5, t(29)=2.00, p>.05]$ and both were above 
new [lag 1, $t(29)=4.90, p<.001 ; \operatorname{lag} 5, t(29)=3.31$, $p<.005]$. A new result was that there was no difference between FA rates to lag 1 conjunctions and lag 5 conjunctions; $t<1$ upright, $t<1$ inverted.

We then compared upright faces across experiments, to ask whether encoding faces singly at study, rather than in pairs, produced a stronger sensitivity to wholeface matches. This was not the case. Percentage "old" responses were very similar for both old $(67.4 \%$ Experiment 1, 67.2\% Experiment 2$)$ and new $(38.2 \%, 35.0 \%)$, indicating that conjunction scores could be directly compared. Averaging the two conjunction conditions in each experiment then produced the same result for each experiment $(52.2 \%, 54.03 \%)$. This argues that Reinitz's use of simultaneous presentation at study was not a major factor in his findings of weak memory for the relationship between parts.

\section{DISCUSSION}

Across both experiments, upright face stimuli were judged as old, to some extent, if their component feature sets were old (conjunction > new), and more so again if the pairing relationship between these parts was also old (old substantially greater than conjunction). Inverted stimuli, in contrast, were judged as old entirely on the basis of component matches, with little access to which eyes-eyebrows had been joined with which nose-mouth at study (old $=$ conjunction $>$ new).

\section{A Face-Processing Theory of Memory Conjunction Errors}

There is general acceptance in the face literature that configural/holistic processing occurs for upright faces (see, e.g., Maurer et al., 2002; but see Wenger \& Ingvalson, 2002). Our results can be naturally interpreted within this framework, by presuming that configural/holistic processing is the mechanism supporting memory for the pairing between parts in the memory conjunction paradigm, and that independent part-based processing supports memory for local component matches.

On this presumption, the present study was motivated by an important concern with the Reinitz memory conjunction studies (Hannigan \& Reinitz, 2000; Reinitz \& Hannigan, 2001; Reinitz et al., 1992) - namely, that these appeared to indicate little or no configural processing for upright faces. In the context of our own results, however, we suggest this unusual finding was probably due to Reinitz's choice of stimuli: (Part-based) generic object processing rather than (configural) face processing can be activated by using unnatural line drawings, attentionattracting hair, and conjunctions formed across hair and internal face regions. Because our own experiment used natural faces with conjunctions made entirely of internal face features, normal face-recognition mechanisms were engaged, producing a pattern of memory responses consistent with configural processing for upright but not for inverted faces. Thus, overall, both our results and Reinitz's can be explained neatly within standard face theories, by making the simple (and not uncommon) observation that it is possible to weight two different perceptual systems differently, depending on the circumstances of the stimuli and/or task.

\section{Memory-Only Theories of Memory Conjunction Errors}

Our explanation of the present results differs considerably from previous explanations of memory conjunction errors; these have been developed in the context of theories of memory, with no reference to perceptual processing. Are there memory-only theories that can provide an explanation? We suggest not.

Within the specific context of explaining memory conjunction errors, three major theories have been discussed (Jones \& Jacoby, 2001; Reinitz \& Hannigan, 2001; Rubin et al., 1999). Our results do not distinguish between these, but they provide an important critique for all. Their shared assumption is that memory-derived theories can be developed independently of the particular type of stimulus tested (e.g., words, sentences, or faces). Our finding of different patterns for upright and inverted faces is contradictory to this idea that stimulus type is irrelevant.

Another theoretical possibility comes from formal memory models. These models could potentially explain our main finding (old $>$ conjunction for upright faces but old = conjunction for inverted faces), in terms of poorer old-new discriminability for inverted (old $=60 \mathrm{vs.} \mathrm{new}=$ 41, Experiment 2 ) than for upright (old $=67$ vs. new $=35$ ): Such models have been shown to predict the reduced difference between hit rate for intact word pairs and FA rates for rearranged pairs, as overall discriminability reduces (e.g., Clark \& Shiffrin, 1992). However, this idea cannot explain the full pattern of findings across studies. For example, Reinitz and Hannigan (2001) found only a small difference between old and conjunction in the context of excellent overall discriminability (old $=79 \%$ vs. new $=15 \%$ ). It also does not explain why memory discriminability for inverted faces was poorer in the first place, given that upright and inverted stimuli were physically identical.

We thus argue that a full theory must make reference to both the nature of perceptual processing and memory factors. The different patterns for upright and inverted are most naturally explained as configural versus partbased perceptual processing effects. However, one result presumably arising from properties of memory was our higher FAs for SCs than for NSCs in Experiment 1. This apparent "proximity" effect was in fact not a temporal proximity effect per se, given that there was no difference between lag 1 and lag 5 conjunctions in Experiment 2. However, it seems reasonable (Reinitz \& Hannigan, 2001) that items that appeared together at study are to some extent coded as part of the same "event," and are thus more confusable in memory than are items on different trials coded as distinct events. 


\section{REFERENCES}

Buxbaum, L. J., Glosser, G., \& Coslett, H. B. (1999). Impaired face and word recognition without object agnosia. Neuropsychologia, 37, 41-50.

Clark, S. E., \& Shiffrin, R. M. (1992). Cuing effects and associative information in recognition memory. Memory \& Cognition, 20, 580-598.

Cohen, J. D., MacWhinney, B., Flatt, M., \& Provost, J. (1993). PsyScope: An interactive graphic system for designing and controlling experiments in the psychology laboratory using Macintosh computers. Behavior Research Methods, Instruments, \& Computers, 25, 257-271

Cohen, L. B., \& CAshon, C. H. (2001). Do 7-month-old infants process independent features or facial configurations? Infant \& Child Development, 10, 83-92.

Hannigan, S. L., \& Reinitz, M. T. (2000). Influences of temporal factors on memory conjunction errors. Applied Cognitive Psychology, 14, 309-321.

Jones, T. C., \& JACOBY, L. L. (2001). Feature and conjunction errors in recognition memory: Evidence for dual-process theory. Journal of Memory \& Language, 45, 82-102.

Kanwisher, N., McDermott, J., \& Chun, M. M. (1997). The fusiform face area: A module in human extrastriate cortex specialized for face perception. Journal of Neuroscience, 17, 4302-4311.

Macmillan, N. A., \& Creelman, C. D. (1991). Detection theory: A user's guide. Cambridge: Cambridge University Press.

Maurer, D., Le Grand, R., \& Mondloch, C. J. (2002). The many faces of configural processing. Trends in Cognitive Sciences, 6, 255-260.
Moscovitch, M., Winocur, G., \& Behrmann, M. (1997). What is special about face recognition? Nineteen experiments on a person with visual object agnosia and dyslexia but normal face recognition. Journal of Cognitive Neuroscience, 9, 555-604

REINITZ, M. T., \& HaNNIGAN, S. L. (2001). Effects of simultaneous presentation and attention switching on memory conjunction errors Journal of Memory \& Language, 44, 206-219.

Reinitz, M. T., Lammers, W. J., \& Cochran, B. P. (1992). Memoryconjunction errors: Miscombination of stored stimulus features can produce illusions of memory. Memory \& Cognition, 20, 1-11.

Rubin, S. R., Van Petten, C., Glisky, E. L., \& Newberg, W. M. (1999) Memory conjunction errors in younger and older adults: Event-related potential and neuropsychological data. Cognitive Neuropsychology, 16, 459-488.

Searcy, J. H., Bartlett, J. C., \& Memon, A. (1999). Age differences in accuracy and choosing in eyewitness identification and face recognition. Memory \& Cognition, 27, 538-552.

TANAKA, J. W., \& FARAH, M. J. (1993). Parts and wholes in face recognition. Quarterly Journal of Experimental Psychology, 46A, 225-245.

Tong, F., \& NAKAYAMA, K. (1999). Harvard Vision Lab Face Database. Cambridge, MA: Harvard University, Vision Lab.

Wenger, M. J., \& IngValson, E. M. (2002). A decisional component of holistic encoding. Journal of Experimental Psychology: Learning, Memory, \& Cognition, 28, 872-892.

Young, A. W., Hellawell, D., \& Hay, D. C. (1987). Configurational information in face perception. Perception, 16, 747-759.

(Manuscript received September 3, 2004; revision accepted for publication June 7, 2005.) 